\title{
Significant Improvement in Shoulder Function and Pain in Patients Following Biologic Augmentation of Revision Arthroscopic Rotator Cuff Repair Using an Autologous Fibrin Scaffold and Bone Marrow Aspirate Derived From the Proximal Humerus
}

\author{
Andreas Voss, M.D., Mary Beth McCarthy, B.S., Nicholas Bellas, M.D., Ralf Kellner, Ph.D., \\ Knut Beitzel, M.D., Felix Dyrna, M.D., Andreas B. Imhoff, M.D., \\ Augustus D. Mazzocca, M.S., M.D., Lukas N. Muench, M.D., and Daniel P. Berthold, M.D.
}

\begin{abstract}
Purpose: To clinically evaluate patients who underwent a biologic augmentation technique in revision arthroscopic rotator cuff repair using an autologous fibrin scaffold and concentrated stem cells isolated from bone marrow aspirate (BMA) obtained from the proximal humerus. Methods: This is a retrospective review of prospectively collected data from patients who underwent biologic augmentation of revision arthroscopic rotator cuff repair using an autologous fibrin scaffold and BMA obtained from the proximal humerus between 2014 and 2015. Minimum follow-up was 12 months. Outcome measures were collected preoperatively and postoperatively including range of motion as well as American Shoulder and Elbow Surgeons Shoulder Form, Simple Shoulder Test, single assessment numeric evaluation, and visual analog score. In addition, BMA samples of each patient were assessed for the number of nucleated cells and colony-forming units. Regression analysis was performed to investigate whether the number of nucleated cells and colony-forming units had an influence on outcome and failure. Results: Ten patients who underwent biologic augmentation of revision arthroscopic rotator cuff repair using an autologous fibrin scaffold and concentrated BMA obtained from the proximal humerus between 2014 and 2015 were included. The mean follow-up time was 30.7 (range: 12-49) months. Four patients were revised at final followup. Postoperative clinical scores improved significantly: American Shoulder and Elbow Surgeons (28.1 \pm 5.4 to 60.9 \pm 9.0; $P<.01)$, single assessment numeric evaluation $(6.6 \pm 2.3$ to $65.1 \pm 10.9 ; P<.01)$, visual analog scale $(7.2 \pm 0.9$ to $3.1 \pm 0.9$; $P<.01)$, and Simple Shoulder Test $(1.6 \pm 0.5$ to $10.3 \pm 5.7 ; P<.01)$. Postoperative range of motion increased significantly with regard to flexion $(97.0 \pm 13.6$ to $151.0 \pm 12.2 ; P<.01)$ and abduction $(88.0 \pm 14.0$ to $134.0 \pm 15.1 ; P=.038)$ but not with external rotation $(38.0 \pm 5.7$ to $50.5 \pm 6.5 ; P=.16)$. Less pain was correlated to an increased number of nucleated cells $(P=.026)$; however, there was no correlation between failure rate and number of nucleated cells $(P=.430)$. Conclusions: Patients who underwent biologic augmentation of revision arthroscopic rotator cuff repair using an autologous fibrin scaffold and concentrated BMA demonstrated a significant improvement in shoulder function along with reduction of pain. However, the overall revision rate for this procedure was $40 \%$. Level of Evidence: Level IV, therapeutic case series.
\end{abstract}

From the Department of Trauma Surgery, Regensburg University Medical Center, Germany (A.V.); Department of Orthopaedic Sports Medicine, Technical University of Munich, Germany (A.V., K.B., A.B.I., L.N.M., D.P.B.); Sporthopaedicum, Regensburg/Straubing, Germany (A.V.); Department of Orthopaedic Surgery, University of Connecticut Health Center, Farmington, Connecticut, U.S.A. (M.B.M., N.B., L.N.M., D.P.B.); Department of Quantitative Methods and Statistics, Saarland University, Saarbrücken, Germany (R.K.); Department of Shoulder Surgery, Arthroscopy and Orthopaedic Sports Medicine, ATOS, Orthoparc Clinic, Cologne, Germany (K.B.); and Department of Trauma, Plastic and Reconstructive Surgery, University of Münster, Germany (F.D.).

The authors report the following potential conflicts of interest or sources of funding: A.D.M. reports research grants from Arthrex. K.B. is a consultant for Arthrex and receives royalties from Arthrex. A.V. is a consultant for DJO Global. The University of Connecticut Health Center / UConn Musculoskeletal Institute has received direct funding and material support for this study from Arthrex Inc. (Naples, Florida, U.S.A.). The company had no influence on study design, data collection or interpretation of the results or the final manuscript. Further conflict of interest statements can be found in the attached files. Full ICMJE author disclosure forms are available for this article online, as supplementary material.

The study was performed at the University of Connecticut, Farmington, Connecticut, U.S.A.

Received March 21, 2021; accepted August 18, 2021.

Address correspondence to Dr. Andreas Voss, M.D., Department of Trauma Surgery, Regensburg University Medicla Center, Germany. E-mail: andreas. voss@ukr.de

(C) 2021 THE AUTHORS. Published by Elsevier Inc. on behalf of the Arthroscopy Association of North America. This is an open access article under the CC BY-NC-ND license (http://creativecommons.org/licenses/by-nc-nd/4.0/). 2666-061X/21402

https://doi.org/10.1016/j.asmr.2021.08.009 
$\mathbf{W}$ hen approaching arthroscopic rotator cuff (RC) surgery, high failure rates, varying between $15 \%$ and $80 \%$ of cases, ${ }^{1-4}$ still raise concerns among shoulder surgeons. Of interest, almost $25 \%$ of retears are observed within the first 2 years after surgery ${ }^{5}$; however, $50 \%$ of these patients are still expected to have satisfactory outcomes. ${ }^{6,7}$ Even though the exact etiology of biologic failure leading to retears after RC repair is not yet fully understood, the formation of tendinous tissue has been shown to be a relevant problem. ${ }^{8}$

As the continuous evolvement of arthroscopic techniques has led to excellent biomechanical properties with regard to tendon-to-bone fixation, recent research has focused on improving RC healing with biological augmentation. $^{8}$ Various ideas have been proposed to aid and potentially improve the limited endogenous healing potential of the RC tissue including the use of concentrated stem cells isolated from bone marrow aspirate (CBMA). ${ }^{9}$ As such, various techniques, and harvest sites, including the proximal humerus, have been demonstrated to be a reliable source of cBMAs for biologic augmentation during arthroscopic surgery. ${ }^{10,11}$ Recently, Hernigou et al. ${ }^{9}$ showed a significant improvement in healing in primary $\mathrm{RC}$ repair by using bone marrow concentrated mesenchymal stromal cells (MSCs) obtained from the iliac crest. In addition, when compared with RC repair alone, a reduced failure rate after 10 years was demonstrated ( $13 \%$ vs $56 \%$ ).

However, when compared with the proximal humerus, harvesting cBMA from the iliac crest may be limited to possible donor-site morbidity, as the harvest location for bone marrow aspiration from the humerus is the one used for anchor placement. ${ }^{12}$ Interestingly, a recent investigation from Otto et al. ${ }^{13}$ showed that samples of BMA harvested from the proximal humerus yielded a significantly greater amount of colonyforming units (CFUs) derived from BMA when compared with samples of BMA obtained from the ilium. In addition, when it comes to biologic augmentation in RC repair, delivery of biologic adjuvants can be challenging. Various techniques ${ }^{14-16}$ have been described; however, scaffolds often are noted to be expensive, tend to loosen, or may float away. Finally, when using biologic augmentation of arthroscopic RC repair, the current literature is focused on primary repair, as the high-failure rate in revision surgery may put this technique beyond the confidence of a shoulder surgeon.

The purpose of this study was to clinically evaluate patients who underwent a biologic augmentation technique in revision arthroscopic RC repair using an autologous fibrin scaffold and cBMA obtained from the proximal humerus. The authors hypothesized patients who underwent biologic augmentation of revision arthroscopic RC repair using an autologous fibrin scaffold and cBMA would have a significant improvement in shoulder function at a minimum 1-year followup.

\section{Methods}

A retrospective review of prospectively collected data from an institutional shoulder registry was performed. All patients included were older than 18 years of age and undergoing revision arthroscopic RC repair augmented using an autologous fibrin scaffold and cBMA harvested from the proximal humerus. Surgeries were performed by a single, shoulder fellowship-trained surgeon (A.D.M.), from January 2014 to March 2015. Institutional review board approval was obtained before initiation of the study by the University of Connecticut (institutional review board \#06-577-2). Patients were included regardless of socioeconomic factors or comorbidities. Patients with less than 1 year of follow-up, RC tear arthropathy (Hamada grade $>3$ ), irreparable massive tears, previous RC surgery requiring tendon transfers, nerve injuries, preoperative pseudoparalysis, or fatty infiltration greater than Goutallier grade III on magnetic resonance imaging (MRI) were excluded from the study. All alternative treatment options were discussed with the patient, including continued conservative treatment. Basic demographic information (age, sex, and body mass index) as well as a thorough medical and surgical history were obtained for each patient.

\section{Clinical and Functional Outcomes}

Objective and subjective outcome measures including the American Shoulder and Elbow Surgeons (ASES) Score, ${ }^{17}$ Constant-Murley score, ${ }^{18}$ single assessment numeric evaluation score, ${ }^{19}$ Simple Shoulder Test, ${ }^{20}$ the visual analog scale for pain, and range of motion in terms of active flexion, abduction, and external rotation were collected preoperatively and at terminal follow-up with a minimum follow-up of 1 year (A.D.M.) Pain scores were obtained from the ASES survey. Previous studies have confirmed these scores in terms of reliability, validity, and responsiveness. ${ }^{19,21-23}$ In addition, the BMA of each patient was assessed for the number of nucleated cells and CFUs. Failure was defined if patients presented with continuous shoulder weakness and pain at minimum follow-up.

\section{Surgical Technique: Autologous Fibrin Scaffold}

Before surgery, all patients received detailed information about the operative technique. All surgeries were performed with the patient in the beach-chair position by a single, shoulder fellowship-trained surgeon (A.D.M.). Following an interscalene block and successful induction of general anesthesia, diagnostic arthroscopy was performed to confirm the presence of 
the RC retear and assess mobility for repair. Loose suture material and/or anchors from previous repairs were removed and the torn RC tendons were mobilized. The surgical technique using an autologous fibrin scaffold for biological augmentation has been described recently. ${ }^{11}$ BMA was obtained from the proximal humerus during arthroscopic RC surgery. For this study, a nonfenestrated trocar was used as it showed a greater capability to extract progenitor cells from the proximal humerus. ${ }^{24}$ The BMA was then transferred to an automated light-absorption system (Angel System; Arthrex, Naples, FL) for cell concentration. For reimplantation of the CBMA, the biologic scaffold consisted of platelet-poor plasma (source of fibrinogen), plateletrich plasma (PRP; source for growth factors), and an external source of bovine thrombin used to activate the clotting cascade. The PRP and platelet-poor plasma preparation was performed as previously described. ${ }^{25,26}$

While the fibrin clot was prepared on the back table by the technical nurse, the surgeon mobilized the RC tendon, placed the medial row suture anchors, and shuttled the medial row sutures through the lateral portal. A modified cannula (Arthrex) was used to shuttle down the fibrin scaffolds onto the footprint, followed by passing the sutures through the tendon followed by placement of the lateral row. This sequence of steps is important to ensure that the scaffold is properly positioned between the RC tendon and the footprint. Finally, an arthroscopic glenohumeral confirmation of the tendon repair was performed to check for scaffold loosening. Tendon repair was performed using a double-row suture bridge (Arthrex). Biceps tenotomy/tenodesis was not required, as all 10 patients had previous RC repair with biceps tenodesis or tenotomy. Subscapularis repair was not needed.

\section{Rehabilitation}

Patients were placed in $30^{\circ}$ abduction sling for a minimum of 6 to 8 weeks with continuous passive and active assisted range of motion. Unrestricted active assisted external rotation and forward elevation was allowed on week 12, and RC muscle strengthening was initiated. Isometric exercises and advance to resisted isotonic exercises were started and continued until postoperative week 18. Focus was set on restoration of scapular stability and strength during the rehabilitation period

\section{Cell Analysis}

One milliliter from each bone marrow aspiration was removed to assess nucleated cell count and the number of CFUs. The cBMA was plated in $100 \mathrm{~mm}^{2}$ Primaria culture dishes (Fisher Scientific, Waltham, MA) and cultured according to a standard protocol. ${ }^{27}$ Cells were placed an incubator at $37^{\circ} \mathrm{C}$ with $5 \% \mathrm{CO}_{2}$. Media was changed after 24 hours and plates were checked every day for the appearance of colonies and contamination. ${ }^{28}$ CFUs were counted after 7 to 10 days, where a CFU was defined as a cluster of cells containing $\geq 8$ cells. Cell analysis was performed by N.B. and supervised by M.M.

\section{Statistical Analysis}

To analyze differences in the expected values between the 2 points in time (time of treatment and time of follow-up), regression analysis was conducted which regresses the variable of interest on a time dummy which is equal to zero at the initial point in time and equal to one at the point of second measurement. The corresponding slope of the regression line represents the difference in the mean between the 2 time points. An estimate that is significantly different from zero speaks for a significant effect of the treatment. The alpha level was 0.05 for all statistical tests, and the analysis was conducted using Stata (StataCorp 2017. Stata Statistical Software: Release 15; StataCorp LLC, College Station, TX).

\section{Results}

In a single surgeon's practice, 10 patients underwent revision RC repair between January 2014 to March 2015 using the autologous fibrin scaffold augmentation and had a minimum 1-year follow-up. The mean age of patients included in this study was $53.6 \pm 9.0$ years (range: 35-64 years) with 3 female and 7 male patients. The mean follow-up time was $30.7 \pm 14.3$ months (range: 12-49 months). Five patients had 1, 3 had 2, and 2 had 3 previous cuff surgeries.

\section{Clinical Outcomes}

Overall, postoperative range of motion increased significantly with flexion $(P<.01)$ and abduction $(P=$ $.04)$ but not with external rotation $(P=.16)$ (Table 1 , Fig 1). Postoperative clinical scores increased significantly (Table 1, Fig 2). In addition, sex and body mass index had no correlation with clinical outcomes (ASES, single assessment numeric evaluation, Simple Shoulder Test, visual analog scale, flexion, abduction and external rotation; all $P>.05$ ).

\section{Biologic Findings and Correlation to Clinical Outcomes}

Data analysis regarding the nucleated cells and number of CFUs only revealed a significant correlation for less pain and increased nucleated cells $(P=.03)$, but no correlation could be found between failure rate and the number of cells $(P=.43)$ (Table 2).

\section{Complications and Revisions}

None of the 10 patients who underwent revision arthroscopic RC repair augmented using the Fibrin-clot technique had intra- or postoperative complications. 
Table 1. Pre- and Postoperative Mean Scores and Range of Motion

\begin{tabular}{|c|c|c|c|}
\hline Score & Preoperative & Postoperative & $P$ Value \\
\hline$\overline{\mathrm{VAS}}$ & $7.2 \pm 0.9$ & $3.1 \pm 0.9$ & $<.01$ \\
\hline SANE & $6.6 \pm 2.3$ & $65.1 \pm 10.9$ & $<.01$ \\
\hline ASES & $28.1 \pm 5.4$ & $60.9 \pm 9.0$ & $<.01$ \\
\hline SST & $1.6 \pm 0.5$ & $10.3 \pm 5.7$ & $<.01$ \\
\hline Active flexion, ${ }^{\circ}$ & $97 \pm 13.6$ & $151.0 \pm 12.2$ & $<.01$ \\
\hline Active abduction, ${ }^{\circ}$ & $88 \pm 14.0$ & $134.0 \pm 15.1$ & .04 \\
\hline $\begin{array}{l}\text { Active external } \\
\text { rotation, }^{\circ}\end{array}$ & $38 \pm 5.7$ & $50.5 \pm 6.4$ & .16 \\
\hline
\end{tabular}

ASES, American Shoulder and Elbow Surgeons; SANE, single assessment numeric evaluation; SD, standard deviation; SST, Simple Shoulder Test; VAS, visual analog scale.

However, 4 patients $(40 \%)$ showed clinical failure and failure on postoperative MRI with weakness and pain.

\section{Discussion}

The most important finding of this study was that $60 \%$ of the patients undergoing revision arthroscopic $\mathrm{RC}$ repair augmented using the Fibrin clot technique achieved significant improvement in functional and clinical outcomes at a minimum l-year follow-up. Although $40 \%$ of the patients showed clinical failure and failure on postoperative MRI with continuous weakness and pain.

Over the past decade, the incidence of arthroscopic RC repairs has increased by almost $600 \%$ with high satisfactory rates being reported. ${ }^{29-31}$ However, retears, which are noted to occur in between $13 \%$ and $80 \%$ of the cases, remain highly challenging for orthopaedic surgeons. To this, almost $25 \%$ of those retears are observed within the first 2 years after surgery, ${ }^{5}$ and are depending on the initial tendon and muscle quality and tear size. ${ }^{3,4}$

Also, of clinical importance, up to $50 \%$ of these patients are presenting with good-to-satisfactory outcomes. ${ }^{6,7}$

The current literature is focused on novel augmentation techniques using biologic adjuvants to support the healing potential of torn tendons, as the endogenous healing potential of torn tendons appears to be limited. ${ }^{13,25,26,32-34}$ These augmentation techniques include the application of growth factors, PRP, or MSCs. ${ }^{9,35}$ As such, bone marrow remains one of the most commonly used sources of MSCs for biological augmentation. Clinically, its application in patients with RC tears results in promising outcomes including decreasing retear rates and improved healing outcomes. ${ }^{9,35,36}$ However, Muschler et al. ${ }^{37}$ recently demonstrated that progenitor cells only averaged about 1 per 30,000 nucleated cells in BMA obtained from the iliac crest. Interestingly, endogenous application of PRP was recently demonstrated to prompt the differentiation of tendon stem cells into active tenocytes, exhibiting high proliferation rates and collagen production capability. ${ }^{38}$

When processing bone marrow from the iliac crest, complications such as hematoma and nerve palsy have been reported. ${ }^{39}$ As such, novel, more safer techniques to obtain bone marrow emerged in recent years. Although aspiration of bone marrow from the iliac crest is still considered the "gold standard," ${ }^{40-43}$ Mazzocca et al. ${ }^{12}$ first described the proximal humerus to be a more desirable source of MSCs for RC repair due to its ease of attainment. The authors stated that the ability to obtain the sample under direct visualization during RC reconstruction makes the proximal humerus an ideal location, even though the proximity of the axillary nerve and artery make the proximal humerus amenable to similar risks. More importantly, BMA concentrate has been shown to contain more growth factors with anti-inflammatory and anabolic potential as well as up to 3 times more nucleated cells when compared with PRP. ${ }^{44}$

Moreover, harvesting and processing BMA concentrate from either the ilium or the humerus is noted to be an expensive, time-consuming procedure with highly debatable cost-effectiveness. ${ }^{45}$ When scanning current literature, only a limited amount of studies with mostly small case series have investigated the effectiveness of biological augmentation using BMA. As such, most of the studies investigated BMA for augmenting single-row RC repairs. ${ }^{9,36,46,47}$ To this, these studies only reported on bone marrow stimulation techniques, rather than direct application of cBMA. ${ }^{9,36,46,47}$ Subsequently, it is still not well understood how these techniques are helping in inducing endogenous RC healing. Also, definite conclusions regarding the clinical efficacy of BMA applications have not been drawn to date. As per Carr and Rodeo, ${ }^{45}$ this is mainly due to inconsistent relationships between successful RC healing and clinical outcomes scores as well as disparities in underlying pathologies, repair techniques, lack of control groups, and patient demographics.

In addition, the current literature focuses on patients requiring primary $\mathrm{RC}$ repair, as revision $\mathrm{RC}$ still remains highly challenging. However, the data from this study show that the fibrin clot technique with cBMA obtained from the proximal humerus may be clinical practicable procedure with no intraoperative complications recorded. However, the high failure rate of $40 \%$ still arises the question, if future invasive procedures such as superior capsular reconstruction or reverse shoulder arthroplasty may be still avoided in this young patient cohort.

\section{Limitations}

There were several limitations to the study. Although the data were collected prospectively, the chart review 


\section{Flexion}

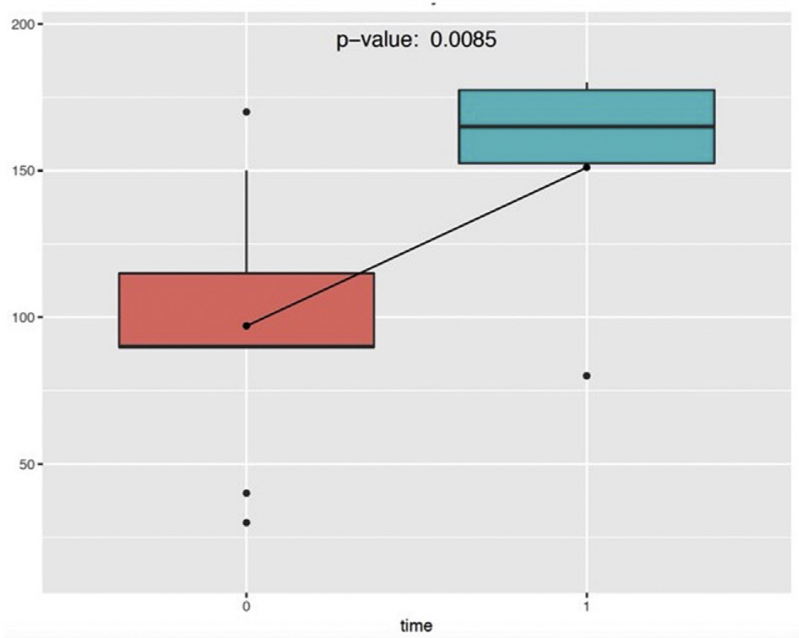

VAS

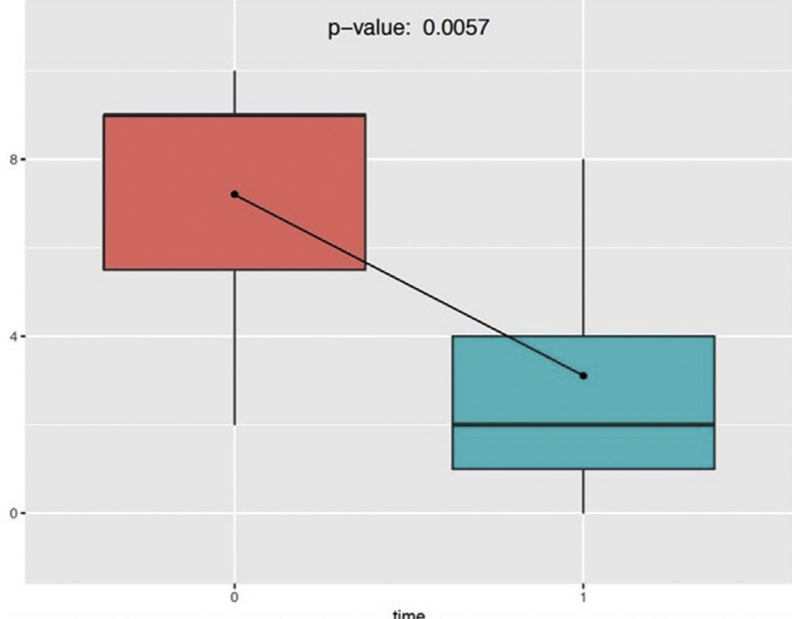

\section{time $0=$ pre-operative time 1 = final follow-up}

Fig 1. Pre- and postoperative range of motion and pain level at final follow-up. VAS, visual analog scale.

was performed retrospectively and may create a selection bias. Also, as this study only reported on outcomes of a single surgeon's practice, external validity may be limited in terms of both patient population and surgical technique. Moreover, the sample size was small; however, this reflects daily clinical practice, as the number of patients undergoing revision RC surgery remains limited. In addition, the large patient-individual variability in harvested biologic adjuvants, including PRP, and cBMA, also may have influenced the results. In addition, this study only reports on preliminary outcomes after a minimum 1-year follow-up; thus, it remains to be seen if patients will maintain significant improvement in shoulder function over a longer-term. Lastly, MRIs or ultrasound scans were not available to evaluate for the status of the repaired tendon and comorbidities were not taken into account when performing the statistical analysis.
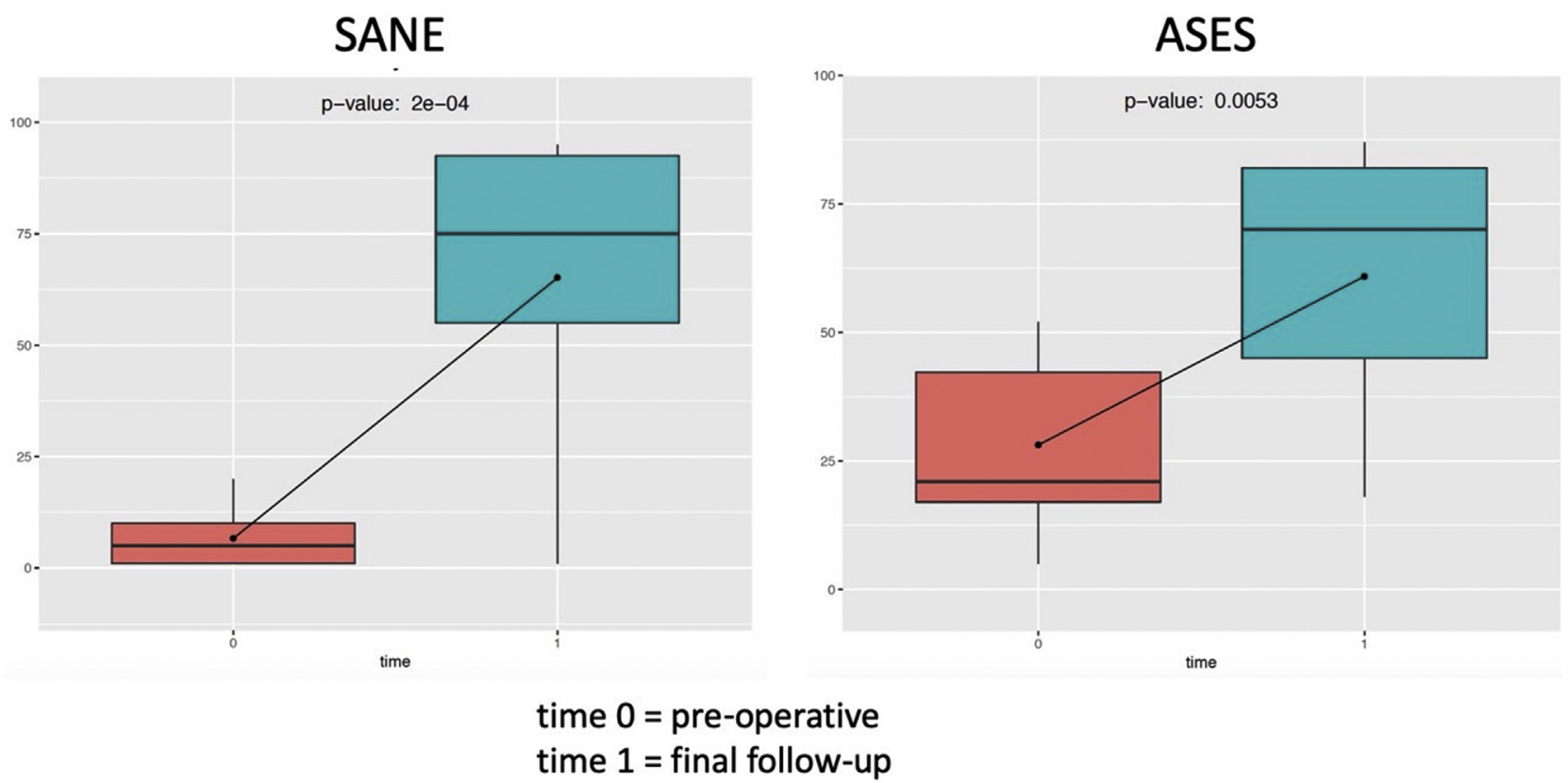

Fig 2. Pre- and postoperative scores at final follow-up. ASES, American Shoulder and Elbow Surgeons; SANE, single assessment numeric evaluation. 
Table 2. Regression Cell Analysis of BMA During Arthroscopic Revision Cuff Surgery

\begin{tabular}{|c|c|c|c|c|c|c|c|c|}
\hline & VAS & SANE & ASES & SST & Failure & $\mathrm{FE}$ & $\mathrm{AB}$ & ER \\
\hline Nucleated cells & $\begin{array}{l}P=.03 \text { (less pain with } \\
\text { increased cells) }\end{array}$ & $P=.17$ & $P=.08$ & $P=.29$ & $P=.43$ & $P=.59$ & $P=.76$ & $P=.33$ \\
\hline CFUs & $P=.85$ & $P=.15$ & $P=.93$ & $P=.98$ & $P=.56$ & $P=.08$ & $P=.36$ & $P=.09$ \\
\hline
\end{tabular}

NOTE. Nucleated cells (mean \pm SD): $23.2 \pm 5.2$ (million/cc BMA).

CFUs (mean \pm SD): $2047.6 \pm 436.8$

$\mathrm{AB}$, abduction; $\mathrm{BMA}$, bone marrow aspirate; $\mathrm{CFU}$, colony-forming unit; ER, external rotation; FE, forward elevation; SANE, single assessment numeric evaluation; SD, standard deviation; SST, Simple Shoulder Test.

\section{Conclusions}

Patients who underwent biologic augmentation of revision arthroscopic RC repair using an autologous fibrin scaffold and concentrated BMA demonstrated a significant improvement in shoulder function along with reduction of pain. However, the overall revision rate for this procedure was $40 \%$.

\section{References}

1. Galatz LM, Ball CM, Teefey SA, Middleton WD, Yamaguchi K. The outcome and repair integrity of completely arthroscopically repaired large and massive rotator cuff tears. J Bone Joint Surg Am 2004;86:219-224.

2. Toussaint B, Schnaser E, Bosley J, Lefebvre Y, Gobezie R. Early structural and functional outcomes for arthroscopic double-row transosseous-equivalent rotator cuff repair. Am J Sports Med 2011;39:1217-1225.

3. Henry P, Wasserstein D, Park S, et al. Arthroscopic repair for chronic massive rotator cuff tears: A systematic review. Arthroscopy 2015;31:2472-2480.

4. Kim I-B, Kim M-W. Risk factors for retear after arthroscopic repair of full-thickness rotator cuff tears using the suture bridge technique: Classification system. Arthroscopy 2016;32:2191-2200.

5. McElvany MD, McGoldrick E, Gee AO, Neradilek MB, Matsen FA III. Rotator cuff repair: published evidence on factors associated with repair integrity and clinical outcome. Am J Sports Med 2015;43:491-500.

6. Namdari S, Donegan RP, Chamberlain AM, Galatz LM, Yamaguchi K, Keener JD. Factors affecting outcome after structural failure of repaired rotator cuff tears. JBJS 2014;96:99-105.

7. Muench LN, Kia C, Williams AA, et al. High clinical failure rate after latissimus dorsi transfer for revision massive rotator cuff tears. Arthroscopy 2020;36:88-94.

8. Apostolakos J, Durant TJ, Dwyer CR, et al. The enthesis: A review of the tendon-to-bone insertion. Muscles Ligaments Tendons J 2014;4:333-342.

9. Hernigou P, Flouzat Lachaniette CH, Delambre J, et al. Biologic augmentation of rotator cuff repair with mesenchymal stem cells during arthroscopy improves healing and prevents further tears: A case-controlled study. Int Orthop 2014;38:1811-1818.

10. Gulotta LV, Kovacevic D, Ehteshami JR, Dagher E, Packer JD, Rodeo SA. Application of bone marrowderived mesenchymal stem cells in a rotator cuff repair model. Am J Sports Med 2009;37:2126-2133.
11. Voss A, McCarthy MB, Allen D, et al. Fibrin scaffold as a carrier for mesenchymal stem cells and growth factors in shoulder rotator cuff repair. Arthrosc Tech 2016;5: e447-451.

12. Mazzocca AD, McCarthy MB, Chowaniec DM, Cote MP, Arciero RA, Drissi H. Rapid isolation of human stem cells (connective tissue progenitor cells) from the proximal humerus during arthroscopic rotator cuff surgery. Am J Sports Med 2010;38:1438-1447.

13. Otto A, Muench LN, Kia C, et al. Proximal humerus and ilium are reliable sources of bone marrow aspirates for biologic augmentation during arthroscopic surgery. Arthroscopy 2020;36:2403-2411.

14. Pandey V, Bandi A, Madi S, et al. Does application of moderately concentrated platelet-rich plasma improve clinical and structural outcome after arthroscopic repair of medium-sized to large rotator cuff tear? A randomized controlled trial. J Shoulder Elbow Surg 2016;25: 1312-1322.

15. Castricini R, Longo UG, De Benedetto M, et al. Plateletrich plasma augmentation for arthroscopic rotator cuff repair: A randomized controlled trial. Am J Sports Med $2011 ; 39: 258-265$.

16. Rodeo SA, Delos D, Williams RJ, Adler RS, Pearle A, Warren RF. The effect of platelet-rich fibrin matrix on rotator cuff tendon healing: A prospective, randomized clinical study. Am J Sports Med 2012;40:1234-1241.

17. Richards R, An KN, Bigliani LU, et al. A standardized method for the assessment of shoulder function. J Shoulder Elbow Surg 1994;3:347-352.

18. Constant CR, Murley AH. A clinical method of functional assessment of the shoulder. Clin Orthop Relat Res 1987;214: $160-164$.

19. Williams GN, Gangel TJ, Arciero RA, Uhorchak JM, Taylor DC. Comparison of the single assessment numeric evaluation method and two shoulder rating scales. Am J Sports Med 1999;27:214-221.

20. Lippitt SB, Harryman DT, Matsen FA. A practical tool for evaluating function: the simple shoulder test. In: Matsen FA, Fu FH, Hawkins RJ, eds. The shoulder: A balance of mobility and stability. Rosemont, IL: American Academy of Orthopaedic Surgeons, 1993;501-518.

21. Beaton D, Richards R. Assessing the reliability and responsiveness of 5 shoulder questionnaires. J Shoulder Elbow Surg 1998;7:565-572.

22. Godfrey J, Hamman R, Lowenstein S, Briggs K, Kocher M. Reliability, validity, and responsiveness of the simple shoulder test: Psychometric properties by age and injury type. J Shoulder Elbow Surg 2007;16:260-267. 
23. Thigpen CA, Shanley E, Momaya AM, et al. Validity and responsiveness of the single alpha-numeric evaluation for shoulder patients. Am J Sports Med 2018;46: 3480-3485.

24. Voss A, McCarthy MB, Singh H, et al. The influence of trocar fenestration and volume on connective tissue progenitor cells (stem cells) in arthroscopic bone marrow aspiration from the proximal humerus. Arthroscopy 2017;33:1167-1174 e1161.

25. Muench LN, Kia C, Berthold DP, et al. Preliminary clinical outcomes following biologic augmentation of arthroscopic rotator cuff repair using subacromial bursa, concentrated bone marrow aspirate, and platelet-rich plasma. Arthrosc Sports Med Rehabil 2020;2:e803-e813.

26. Muench LN, Baldino JB, Berthold DP, et al. Subacromial bursa-derived cells demonstrate high proliferation potential regardless of patient demographics and rotator cuff tear characteristics. Arthroscopy 2020;36:2794-2802.

27. Voss A, McCarthy MB, Hoberman A, et al. Extracellular matrix of current biological scaffolds promotes the differentiation potential of mesenchymal stem cells. Arthroscopy 2016:32:2381-2392.e2381.

28. Beitzel K, McCarthy MB, Cote MP, et al. Rapid isolation of human stem cells (connective progenitor cells) from the distal femur during arthroscopic knee surgery. Arthroscopy 2012;28:74-84.

29. Bedi A, Dines J, Warren RF, Dines DM. Massive tears of the rotator cuff. J Bone Joint Surg Am 2010;92:1894-1908.

30. Brochin RL, Zastrow R, Hussey-Andersen L, Parsons BO, Cagle PJ. Revision rotator cuff repair: A systematic review. J Shoulder Elbow Surg 2019;29:624-633.

31. Collin P, Abdullah A, Kherad O, Gain S, Denard PJ, Lädermann A. Prospective evaluation of clinical and radiologic factors predicting return to activity within 6 months after arthroscopic rotator cuff repair. I Shoulder Elbow Surg 2015;24:439-445.

32. Landry A, Levy BJ, McCarthy MB, et al. Analysis of time to form colony units for connective tissue progenitor cells (stem cells) harvested from concentrated bone marrow aspirate and subacromial bursa tissue in patients undergoing rotator cuff repair. Arthrosc Sports Med Rehabil 2020;2:e629-e636.

33. Morikawa D, Muench LN, Baldino JB, et al. Comparison of preparation techniques for isolating subacromial bursaderived cells as a potential augment for rotator cuff repair. Arthroscopy 2020;36:80-85.

34. Muench L, Berthold D, Kia C, et al. Concentrated bone marrow aspirate and subacromial bursa-derived cells demonstrate similar cellular adhesion and proliferation potential on demineralized bone matrix scaffolds for biologic augmentation of rotator cuff repair. Muscles Ligaments Tendons J 2020;10:48-56.
35. Imam MA, Holton J, Horriat S, et al. A systematic review of the concept and clinical applications of bone marrow aspirate concentrate in tendon pathology. SICOT J 2017;3: 58.

36. Ellera Gomes JL, da Silva RC, Silla LM, Abreu MR, Pellanda R. Conventional rotator cuff repair complemented by the aid of mononuclear autologous stem cells. Knee Surg Sports Traumatol Arthrosc 2012;20:373-377.

37. Muschler G, Boehm C, Easley K. Aspiration to obtain osteoblast progenitor cells from human bone marrow: The influence of aspiration volume. J Bone Joint Surg Am 1997;79:1699-1709.

38. Zhang J, Wang JHC. Platelet-rich plasma releasate promotes differentiation of tendon stem cells into active tenocytes. Am J Sports Med 2010;38:2477-2486.

39. Hernigou J, Picard L, Alves A, Silvera J, Homma Y, Hernigou P. Understanding bone safety zones during bone marrow aspiration from the iliac crest: The sector rule. Int Orthop 2014;38:2377-2384.

40. McLain RF, Fleming JE, Boehm C, Muschler G. Aspiration of osteoprogenitor cells for augmenting spinal fusion: Comparison of progenitor cell concentrations from the vertebral body and iliac crest. J Bone Joint Surg Am 2005;87:2655-2661.

41. Patterson TE, Boehm C, Nakamoto C, et al. The efficiency of bone marrow aspiration for the harvest of connective tissue progenitors from the human iliac crest. J Bone Joint Surg Am 2017;99:1673-1682.

42. Zumstein MA, Ladermann A, Raniga S, Schar MO. The biology of rotator cuff healing. Orthop Traumatol Surg Res 2017;103:S1-S10.

43. Imam MA, Holton J, Ernstbrunner L, et al. A systematic review of the clinical applications and complications of bone marrow aspirate concentrate in management of bone defects and nonunions. Int Orthop 2017;41: 2213-2220.

44. Zhong W, Sumita Y, Ohba S, et al. In vivo comparison of the bone regeneration capability of human bone marrow concentrates vs. platelet-rich plasma. PLoS One 2012;7. e40833-e40833.

45. Carr JB 2nd, Rodeo SA. The role of biologic agents in the management of common shoulder pathologies: Current state and future directions. J Shoulder Elbow Surg 2019;28: 2041-2052.

46. Milano G, Saccomanno MF, Careri S, Taccardo G, De Vitis R, Fabbriciani C. Efficacy of marrow-stimulating technique in arthroscopic rotator cuff repair: A prospective randomized study. Arthroscopy 2013;29:802-810.

47. Taniguchi N, Suenaga N, Oizumi N, et al. Bone marrow stimulation at the footprint of arthroscopic surfaceholding repair advances cuff repair integrity. J Shoulder Elbow Surg 2015;24:860-866. 